\title{
Le rôle des Grammaires destinées aux dames dans la disciplinarisation du français (XVIII ${ }^{e}$ siècle)
}

Javier Suso López

\section{(2) OpenEdition \\ Journals}

Édition électronique

URL : https://journals.openedition.org/dhfles/3129

DOI : $10.4000 /$ dhfles.3129

ISSN : 2221-4038

Éditeur

Société Internationale pour l'Histoire du Français Langue Étrangère ou Seconde

Édition imprimée

Date de publication : 1 juin 2012

ISSN : 0992-7654

Référence électronique

Javier Suso López, «Le rôle des Grammaires destinées aux dames dans la disciplinarisation du français (XVIII siècle) », Documents pour l'histoire du français langue étrangère ou seconde [En ligne], 47-48 | 2012, mis en ligne le 01 janvier 2015, consulté le 03 avril 2023. URL : http:// journals.openedition.org/dhfles/3129; DOI : https://doi.org/10.4000/dhfles.3129

Ce document a été généré automatiquement le 3 avril 2023.

Tous droits réservés 


\title{
Le rôle des Grammaires destinées aux dames dans la disciplinarisation $d u$ français (XVIII ${ }^{\mathrm{e}}$ siècle)
}

\author{
Javier Suso López
}

\section{Introduction}

1 Les grammaires destinées aux dames constituent la manifestation éditoriale d'un vaste phénomène socio-éducatif qui se met en place en Europe à partir du début du XVII ${ }^{\mathrm{e}}$ siècle, de façon particulière dans certains pays, comme les Pays-Bas septentrionaux ou la France : l'éducation des filles. Ce n'est donc pas un hasard si, à la fin du XVII ${ }^{e}$ siècle, plusieurs traités sont édités en France sur cette question : De l'égalité des deux sexes et $D e$ l'éducation des dames pour la conduite de l'esprit dans les sciences et dans les mœurs (Poulain de la Barre, 1673 et 1674); Traité de l'éducation des filles (Fénelon, 1687); Traité du choix et de la conduite des études (abbé Fleury, 1687),destiné à l'éducation de 'Messieurs les princes de Conti', mais qui s'occupe aussi des études pour les femmes au chapitre XXXVIII...

2 Ces traités vont constituer pendant longtemps le fondement du discours sur la matière, sur lequel se grefferont des commentaires, des répliques ou des contestations de toutes sortes : citons notamment, pour notre propos, l'ouvrage de Madame de Lambert (Avis d'une mère à sa fille, 1728). Si le droit des femmes aux études est reconnu par ces auteurs - étant donné qu'elles possèdent une capacité égale à celles des hommes (dérivée de leur «âme » ou de leur raison, selon la formule de Poulain de la Barre : «l'esprit est universel» (1674:296) -, un discours officiel s'installe : de même qu'on ne fournit pas une éducation égale mais différenciée en fonction de l'origine sociale (à quoi bon donner des études latines aux fils de paysans ?), il faut adapter l'éducation des femmes à leur futur rôle dans la société et à leurs 'dispositions naturelles'.

3 Ainsi, dans le domaine linguistique, l'éducation latine n'est pas conseillée : " La grammaire ne consistera, pour elles, qu'à lire et écrire, et composer correctement en 
français une lettre, un mémoire, ou quelque autre pièce à leur usage [...] (Fleury 1687 : 264-270). Ce qui ne veut point dire qu'on leur interdise d'en faire, mais, comme pour le reste des disciplines (histoire, mathématiques, poésie...), elles doivent les entreprendre en tant que "curiosités ", étant donné qu'elles ne vont pas en faire une profession, et que celles-ci vont constituer uniquement des ornements (ibid.: 264-270). Fénelon continue cette même ligne de raisonnement. Les femmes ne doivent pas trop savoir, il ne s'agit pas d'en faire des savantes, car « la curiosité les rend vaines et précieuses » (1883-1909 [1687] : 2)1, et il suffit qu'elles apprennent à lire et à écrire, l'orthographe (1883-1909 [1687]: 129).

4 Par ce biais, et de façon certes paradoxale, l'éducation offerte aux filles va constituer un pôle antithétique qui se dresse face à l'éducation traditionnelle dispensée aux garçons, qui se faisait dans des collèges dépendant des universités ou des congrégations religieuses masculines, dont la base était le latin (notamment chez les jésuites) :

Dans les pensions et les institutions de jeunes filles, le latin n'est jamais enseigné. La base des études est le français, sous l'Ancien Régime comme au XIXe siècle : 'la science fondamentale de l'instruction des jeunes filles est la connaissance de leur langue',

écrit encore l'abbé Le Tellier au XIX ${ }^{e}$ siècle (Édouard Le Tellier, 1866 : 85, in Chervel $2008: 43)$.

6 Ainsi, les expériences éducatives destinées aux femmes comprennent non seulement un enseignement en langue française, mais aussi un enseignement de la langue française elle-même: «le français, sous la forme de la grammaire, de l'orthographe, de la composition, voire de la correspondance commerciale, tient une place importante dans les plans d'éducation qu'affichent les publicités dans la presse locale » (Chervel 2008 : 45). Mais aussi, il s'y constitue des expériences de renouvellement, des 'plans d'étude' (qui s'ouvrent dès l'origine sur les disciplines modernes) et des méthodes d'enseignement (qui commencent à rivaliser avec le modèle régnant de l'éducation linguistique, fortement imprégné par la ratio studiorum des jésuites). De telle sorte que l'éducation des filles se situe sur le vecteur de l'évolution et du progrès, en ce qui concerne l'éducation linguistique. Nous allons essayer de le démontrer au cours de cet article à travers l'examen de quelques ouvrages destinés aux femmes publiés au XVIII siècle $^{2}$.

\section{La Grammaire italienne à l'usage des dames de l'Abbé Antonini (1728)}

7 La Grammaire italienne à l'usage des dames, avec des dialogues et un Traité de la poësie, de l'Abbé Antonini $(1728)^{3}$ s'adresse à ce créneau précis du public: "Au reste, cette grammaire sera principalement à l'usage des Dames » (Préface, 2-3). Antonini a tiré les leçons d'une grammaire italienne composée deux ans auparavant (ouvrage que «la plûpart [...] ont [...] négligé comme trop chargé de Regles \& de recherches », ibid.); il compose sa nouvelle Grammaire pour ceux qui ne savent pas le latin, dont principalement les femmes :

car j'ai travaillé pour ceux qui veulent apprendre sans peine, ou du moins avec une mediocre application. Une simplicité méthodique, \& dénuée de toute érudition, leur plaît, les amuse et les instruit: Une Méthode trop scavante \& trop etudiée les étonne, les rebute \& ne les instruit point. (Préface, 2) 

suivi de «Dialogues» (p. 236-287, "puisqu'on les croit utiles aux commençans»), présentés en version bilingue, en regard (texte italien à gauche, texte français à droite) et qui reprennent les situations de communication habituelles dans les ouvrages des siècles antérieurs; un "Traité de notre poësie" (325-354) complète l'ouvrage. Les composantes de cet ouvrage ne diffèrent pas de celles des grammaires «de langue étrangère » de l'époque (cf. la Nouvelle Méthode pour apprendre facilement et en peu de temps la langue italienne de Claude Lancelot (1659, Paris, Le Petit ${ }^{4}$, ou encore celle pour la langue espagnole, de 1660); ou bien Le Maitre italien ou Grammaire italienne de Veneroni (1678; édition consultée : 1747, Lyon, Bruyset frères). L'enseignement de la langue étrangère se fait en français (comme l'avait institué Lancelot); la présentation grammaticale est réduite aux « Regles les plus nécessaires \& les plus simples, énoncées avec toute la clarté, la précision \& la brièveté »; l'auteur «joint des exemples aux Regles, \& les exemples ne sont que des phrases les plus aisées, \& les plus ordinaires dans la conversation " (Préface, 2) ; le métalangage grammatical y est expliqué ; des dialogues, qui ont pour but de faire acquérir la conversation (habileté d'expression orale) viennent compléter le dispositif pédagogique; enfin, le traité de poésie (qui comprend un choix de poésies italiennes) introduit la beauté de la langue comme composante qui achève la formation linguistique.

Antonini réduit à l'essentiel le contenu grammatical, sans renoncer pour autant ni au métalangage ni à l'« outil » grammatical : il suit le modèle selon lequel l'enseignement de la langue (étrangère ou maternelle), même pour ceux qui ne savent pas le latin, dont les femmes, se fait après l'acquisition d'un métalangage grammatical de base, qui est supposé commun à toutes les langues. Mais, tout en partageant le besoin de grammaire, et en tirant les leçons de l'échec de sa grammaire de 1726, il s'oppose à ceux qui vantent la complétude ou l'intégralité de la description grammaticale comme point fort dans l'acquisition d'une langue. La grammaire d'Antonini comporte en outre un objectif particulier : le choix de poésies italiennes est destiné à former un goût littéraire disons supra- ou inter-linguistique, à partir duquel la composition de poésies françaises (et non pas italiennes) est incorporée aux « pratiques de travail ».

\section{Les grammaires de D.-É. Choffin, M. de Prunay, N. Adam, L. Barthélemy}

L'édition d'ouvrages destinés à l'éducation linguistique des femmes connaît une forte augmentation à partir de la deuxième moitié du XVIII siècle : ils se présentent sous une grande variété de formes (des grammaires, des traités d'orthographe, des rhétoriques, des encyclopédies.... $)^{5}$. Avant de nous occuper des 'grammaires' à proprement parler, nous devons signaler l'existence de deux ouvrages (une rhétorique, de G.-H. Gaillard, et une encyclopédie, d'A.-J. Panckoucke) qui manifestent pleinement l'essor de ce phénomène éditorial.

11 La Rhétorique française à l'usage des jeunes demoiselles de G.-H. Gaillard (12 éditions de 1746 à 1811) montre que le "bel art de parler ", atout pour briller en société, s'est pleinement constitué en objet de désir, donc susceptible d'être soumis au travail et à la formation (voir Préface, aij", 1748). Gaillard destine son ouvrage aux «jeunes personnes, dont le goût naissant \& imparfait n'ayant point encore d'objet certain,

Documents pour l'histoire du français langue étrangère ou seconde, 47-48 | 2012 
pourrait s'égarer malheureusement en suivant des guides infidèles et pernicieux " (1748: Préface, aij ${ }^{\nu}$ ). Lui-même utilise un procédé rhétorique pour gagner les femmes à sa faveur en louant les capacités naturelles des femmes pour la parole...

Les Études convenables aux demoiselles, contenant la grammaire, la poésie, la rhétorique, le commerce des lettres [...] d'André-Joseph Panckoucke $(1759,1773)$ constituent une encyclopédie destinée à l'éducation des filles : on commence par la Grammaire (p. 1-55), la Poétique françoise (p.56-121), la Rhétorique (p.122-202), la rédaction de lettres; viennent ensuite les différentes matières complétant une culture de base. Pour Panckoucke, l'éducation dispensée aux femmes (à travers « des études \& des lectures convenables à leur état ») doit être orientée "à leur former l'esprit \& le cœur »), sans renoncer à la grammaire, «trop négligée parmi les Dames ", mais indispensable pour l'acquisition de l'écriture :

L'Ecriture est donc l'Art d'exprimer par des figures subsistantes le son de la voix, qui périt \& s'envole à mesure qu'elle est prononcée, c'est un portrait de la parole, qui exprime par des traits ce que nous avons à dire, comme la voix exprime par des paroles ce que nous avons pensé; mais l'un \& l'autre portrait doit être vrai et conforme aux règles. Le précis court et simple que nous avons fait de cette science ne fatiguera point, on s'est borné au pur nécessaire. (1759 : Préface)

La Grammaire françoise [...]de David-Étienne Choffin connaît un succès énorme, à en juger par le nombre d'éditions et d'adaptations qui en ont été publiées. L'édition de $1747^{6}$ contient une dédicace adressée «À mesdemoiselles les gouvernantes et autres demoiselles qui enseignent le français en Allemagne ». Dans les éditions de 1755/56 et 1763, la référence faite aux «demoiselles françaises qui enseignent le français en Allemagne » a disparu, au profit de toutes les «Dames». On constate qu'un public féminin désireux d'apprendre le français s'est constitué en Allemagne, avec une force suffisante pour devenir un créneau rentable pour des éditeurs. Choffin ne ferme pas la porte à d'autres types de destinataires (tous les commençants, Discours préliminaire, $1757: 8$ ), mais il affirme nettement que c'est « en particulier pour ces personnes-là [les dames] qu'elle a été composée » $(1757: 374)$.

Choffin adapte les composantes de sa Grammaire aux «besoins de formation » des dames et à leurs caractéristiques (des « commençans»). Il introduit ainsi la table des souverains de l'Europe et spécialement des princesses de l'Empire, les formes de politesse à utiliser, des tableaux synoptiques (grammaire « réduite en tables»)... Mais c'est dans le tome II, « Renfermant, outre plusieurs choses qui regardent la pratique de la grammaire, diverses pièces qui ont paru propres à former le style, l'esprit et le cœur ", où Choffin innove de façon décidée. Au long de 454 pages, Choffin propose une panoplie d'éléments langagiers et de lectures destinés à pourvoir à l'éducation linguistique et culturelle d'une jeune demoiselle de la classe bourgeoise ou de l'aristocratie :

1. des catalogues de façons de parler adaptées aux diverses situations de communication propres au mode de vie des dames du monde : compliments, pour prendre congé, pour faire visite, manières 'civiles et honnêtes' de s'exprimer, de contredire...

2. des éléments langagiers destinés à parfaire le style, tirés de la Gazette de Leide

3. des modèles de lettres accompagnés d'un traité « sur la façon de composer des lettres »

4. des règles de bienséance

5. des lectures morales

6. des contenus culturels de toutes sortes (histoire, géographie, merveilles du monde) 
7. des sujets de conversation à la mode, précieux, anodins, mais nécessaires: pensées ingénieuses (pour briller en public)...

8. des sujets de l'intérêt des femmes : les vestales, les sibylles, les fées...

9. des biographies des dames illustres (anciennes, modernes, de l'ancien et du nouveau Testament, de la France ou de l'Angleterre...)

10. un choix de poésies et une sélection de fables (en prose et en vers)...

Il inclut également la 'Lettre de Madame la Marquise de Lambert à sa fille sur la véritable éducation' (192-214) et l'Instruction d'un père à sa fille, par Monsieur Dupuy' (215-223). La vie de dames célèbres complète le volume.

Remarquons qu'il s'agit de donner aux dames une formation linguistique complète : la formule «le style, le goût et le cœur " comprend ainsi les habiletés langagières, la composition de lettres et/ou de poésies, le goût poétique, le goût de la lecture, mais aussi la volonté de se surpasser, la conscience du rôle de la femme...

La Grammaire des dames, où l'on trouvera des principes sûrs et faciles pour apprendre à ortographier correctement la langue française, avec les moyens de connaitre les expressions provinciales, de les éviter et de prévenir chez les jeunes demoiselles l'habitude d'une prononciation vicieuse... par M. de Prunay (1777), révèle un fait important : l'expression "grammaire des dames" est établie, en tant que label définitoire d'un type de grammaire. Remarquons cependant que l'objectif de formation est nettement réduit en rapport aux ouvrages antérieurs: ce n'est pas l'«écriture " qui est ciblée, mais l'orthographe, savoir-faire central auquel on ajoute le bon choix des expressions et la prononciation. L'objet des Remarques de Marguerite Buffet (1668) est repris, en l'adaptant à l'évolution de la langue produite et aux règles d'orthographe et de prononciation établies par l'Académie française.

La vraie manière d'apprendre une langue quelconque vivante ou morte par le moyen de la langue française, de Nicolas Adam (1779), annonce sur la page de titre un projet éditorial ambitieux : tout d'abord, une « Grammaire françoise, à l'usage des Dames, servant de base à toutes les autres langues [...]», qui constitue le premier volume; seront éditées par la suite une grammaire latine (1786, seconde édition ${ }^{7}$, qui a comme complément les Quatre chapitres, 1780), une grammaire italienne (1783, qui contient les Fables de Phèdre, en version bilingue) et une grammaire anglaise (1783) ; l'ensemble sera complété par un dernier ouvrage, publié en 1787 , intitulé La vraie manière d'apprendre une langue quelconque vivante ou morte par le moyen de la langue française. Démonstration et pratique de la nouvelle méthode d'enseignement. N. Adam institue une méthode (dans le sens traditionnel de cheminement, qui comprend autant le contenu que la façon de procéder) pour apprendre la langue française : il s'agit en fait de la technique de la traduction interlinéaire de Dumarsais et Radonvilliers améliorée ou complexifiée ( $c f$. Besse 1996, et Reuillon-Blanquet 1994) sous l'appellation de "traduction sous quatre faces ". Son intérêt dépasse toutefois ce fait, et Adam matérialise aussi une idée (déjà présente chez Comenius [1648] 2005: chapitre XXI) selon laquelle, à partir de la connaissance consciente (donc grammaticale) d'une langue - pour Comenius, le latin ; pour N. Adam, une " petite Grammaire nationale, purement élémentaire ", puisque les grammaires de toutes les langues participent à des principes et des caractéristiques universels - on peut accéder à la connaissance d'autres langues par la voie de transpositions et de comparaisons. Sa grammaire est ainsi une "méthode de la Grammaire Françoise universelle à l'usage des Dames", ou, en plus bref, une "grammaire française, à l'usage des Dames". On constate que l'expression 
"grammaire des dames" a changé de sens: c'est d'une grammaire simplifiée qu'il s'agit pour N. Adam, valable pour la "grammaticalisation" de n'importe quel commençant. Disons encore que N. Adam ne prend pas comme base de la pratique de la langue des dialogues courants, mais des textes littéraires classiques: il simplifie la description de la langue, mais il ne renonce point à l'acquisition des objectifs de formation linguistico-littéraire (« le style, le goût, le cœur »).

La 'nouvelle méthode' de N. Adam juxtapose ainsi de façon paradoxale deux réalités habituellement séparées : une grammaire simplifiée (destinée à un public non scolarisé) et les textes littéraires de l'antiquité gréco-latine (réservés jusque-là à des élèves qui suivaient le cursus des collèges); il prétend ainsi étendre la formation littéraire à une couche de population très large, dont les femmes. Pour Adam, la lecture des textes littéraires 'classiques', élevés par la critique au Parnasse, ne doit pas être réservée aux institutions qui offrent une éducation réglée (collèges) pour les seuls garçons, mais ces textes peuvent être lus par tous, y compris les jeunes filles, qui composent l'essentiel des effectifs de ses cours privés.

Louis Barthélemy, qui compose une Grammaire des dames ou nouveau traité d'orthographie françoise : réduite aux regles les plus simples, en 1785, édite également trois ans plus tard La cantatrice grammairienne ou l'art d'apprendre l'orthographe seul, sans le secours d'un maitre, par le moyen des chansons... : avec un portrait des poëtes chansonniers les plus agréables de notre nation et un modele de lettres mêlées de réflexions sur le type épistolaires: ouvrage destinées aux dames, ouvrage absolument hors-pair dans la bibliographie de l'enseignement de la grammaire, et non seulement aux Dames, mais de façon absolue.

Nous n'offrirons ici que quelques caractéristiques qui nous ont mené à cette qualification. L'auteur lui-même dresse l'originalité de son ouvrage dans la Préface :

Présenter à la fois aux Dames un Modele de Lettres, de nouvelles réflexions sur le style épistolaire, le portrait de nos poëtes les plus agréables, \& un chansonnier qui leur fournit les moyens d'apprendre seules, sans le secours de nos maittres, l'orthographe françoise ; voilà tout le mérite de cet ouvrage. (1788 : Préface, I)

\section{Et aussi :}

Nous pouvons dire, sans rien hasarder, que le plan \& les moyens de l'ouvrage que nous offrons à ce sexe aimable, n'avoient jamais été conçus. Et quel est ce plan? quels sont ces moyens? L'art d'apprendre seul, sans le secours des maîtres, l'Orthographe françoise, par le moyen des chansons érotiques, pastorales, villageoises, etc. $(1788: \mathrm{X})$

Si nous regardons le contenu de formation (grammaticale et linguistique) de $\mathrm{La}$ Cantatrice grammairienne, les moyens mis en place ne ciblent pas uniquement l'orthographe (qui possède une place importante, il est vrai: p. 108-303), mais l'ensemble des parties du discours (p. 1-107); l'ouvrage contient aussi un « modèle des lettres mêlées de Réflexions sur le style épistolaire » (p. 304-414). Ce contenu est étayé d'un savoir littéraire spécifique («[le] Portrait des Poëtes chansonniers les plus agréables de notre nation", page de titre). Si les connaissances grammaticales sont incontournables, elles se réduisent à l'essentiel, en éliminant les complexités terminologiques, en reprenant le métalangage de base sous forme de définitions notionnelles simples et en fournissant d'abondants exercices d'application à l'aide desquels fixer ces notions.

C'est surtout l'idée de faire apprendre la langue française au moyen de chansons qui nous semble extraordinaire : il propose un chansonnier, ou un catalogue de chansons 
de toutes sortes : érotiques, pastorales, villageoises ; c'est-à-dire, des poésies récentes mises en musique et des chansons traditionnelles (villageoises). L'adjectif 'érotique' ne doit pas conduire à erreur : ce sont des poésies qui parlent de l'amour, des sentiments affectifs et du plaisir d'aimer, tout simplement ${ }^{8}$. Barthélemy justifie son choix par la prédisposition innée des femmes à la musique (stéréotype récurrent): "L'humeur chansonniere, en général, est un des caracteres des femmes; tristes ou gaies, elles chantent toujours ; \& l'on dirait que la chanson est l'expression naturelle de tous leurs sentiments » (Préface, XI). Au-delà des stéréotypes sur les dispositions naturelles des femmes (aspect que N. Minerva met en relief, 2000: 78-80 et 96-97), le recours aux chansons devient un atout central de sa démarche, car « il est agréable d'apprendre sa langue en chantant » (ibid.). Barthélemy revendique ainsi le plaisir d'apprendre, face au châtiment corporel, aux punitions sévères, à la discipline aveugle..., qui faisaient partie du "programme" d'enseignement des écoles et des collèges des garçons, et qui constituaient le moyen d'affermir leur caractère, d'en faire des hommes. Installer le principe du plaisir dans l'éducation était ainsi un programme révolutionnaire, dont participe Barthélemy, même s'il le réserve aux femmes.

\section{En guise de conclusion}

Ce survol ne permet point d'arrêter des conclusions, mais plutôt d'avancer une série d'idées ou de pistes à étayer par des recherches plus précises. Du point de vue historico-linguistique (histoire de la langue française, histoire de la grammaire), nous ne pouvons qu'être d'accord avec les conclusions proposées par N. Minerva (2000: 102) : les grammaires des dames du XVIII e siècle ne constituent point un «type » de grammaire qui pourrait être défini par rapport aux destinataires (sauf celle de Choffin); elles sont des «testi che fanno poco spazio alla concettualizazione grammaticale per privilegiare il repertorio delle forme, le esemplificazione abbondanti e l'esercizio mnemonico... ». Mais d'un point de vue socio-linguistique et socio-culturel, cette «etichetta [...] apposta a manuali elementari » manifeste une transformation profonde des consciences, au sujet de l'éducation des femmes mais aussi au sujet de l'éducation linguistique (pour toute la population ayant le français comme langue maternelle). À cet égard, il est intéressant de connaître (entre autres) l'étude de C. Pellandra, qui retrace certaines composantes du «climat » ou de l'« atmosphère » dans lesquels se situent ces ouvrages (2000: 103-115).

Les "grammaires destinées aux dames » ne visent pas uniquement l'acquisition d'une correction linguistique (dans l'orthographe, la prononciation, les façons de parler...). Cette formation de base, d'ordre pratique, se complète par d'autres composantes: des habiletés langagières - concernant notamment les qualités de la conversation (cf. Gaillard) -, mais aussi, la formation du goût littéraire, donc, l'accès à la beauté du langage (l'élégance : le « style »), et, au-delà, la formation de « l'esprit » (l'intelligence) et $d u$ « cœur» (pour reprendre les termes de Choffin). Nous pouvons ainsi confirmer les constatations déjà effectuées par M. Reuillon-Blanquet (1995: 64). L'acquisition des compétences langagières et du goût littéraire s'accompagne donc d'une série de valeurs morales, personnelles et sociales et/ou civiques (le savoir-vivre en société), qui sont transmises dans les textes de lecture proposées (anecdotes, histoires, textes littéraires classiques, essais et réflexions, chansons, poésies, modèles de lettres). Bien sûr, nous sommes dans un cadre élitaire : ce sont les usages de la langue de haut niveau qui sont 
visés (la conversation, le style épistolaire, le goût littéraire, les normes sociales et les sujets de conversation polie et galante).

Aussi, les "grammaires des dames " ont procédé à une substitution du latin par le français dans la formation linguistique, rendant possible (et réelle) non seulement une éducation en français, mais aussi l'étude de la langue française elle-même de la part d'une nouvelle couche de la population : c'est à travers le français que les 'dames' bientôt, les 'filles' - et tous ceux qui ne savent pas le latin acquièrent une formation concernant les notions grammaticales; et c'est à travers des textes littéraires en français (au sens large de l'époque) qu'est assuré le rôle dévolu aux "humanités classiques » (dans l'éducation en latin produite dans les collèges religieux ou dépendant des universités).

Le rôle des grammaires des dames consiste ainsi à avoir contribué à créer dans les consciences des contemporains un « imaginaire linguistique " nouveau, à partir d'une série d'éléments idéologiques et de pratiques qui bouleversent la culture scolaire de l'époque et qui produisent une faille importante dans le système. En effet, ce modèle alternatif d'éducation linguistique manifeste :

1.tout d'abord, le «besoin » de la mise en place de l'éducation des femmes: des progrès importants dans la satisfaction de cette demande se réalisent dans ce sens le long du XVIII ${ }^{\mathrm{e}}$ siècle. Bien sûr, il s'agit d'une éducation encore réduite à certaines couches sociales; bien sûr, en correspondance à leur futur rôle dans la société et à leurs 'dispositions naturelles' : ce sera néanmoins la porte d'entrée pour qu'elles démontrent que leurs dispositions naturelles sont égales à celles des hommes et que l'éducation s'étende à toutes les femmes ;

2. l'éducation de la langue française et à travers la langue française - déjà acquise dans certains collèges religieux, et pratiquée également dans toute l'éducation particulière ainsi que dans de nombreuses institutions privées ( $c f$. Chervel 2008: 43-47) - va être partagée comme désirable par l'ensemble de la société et pour tous les enseignements ;

3. la place centrale réservée aux textes « littéraires». Leur statut change de nature : ils ne sont plus réduits au rôle de support matériel d'un exercice linguistico-grammatical (préalable: sous forme de lecture versionnelle; ou postérieur : lieu d'application), mais ils deviennent le lieu même où s'exerce l'apprentissage des qualités langagières et des vertus morales ;

4. des pratiques pédagogiques innovantes, qu'on peut globalement caractériser sous la formule d'une « pédagogie souriante ». Reuillon-Blanquet (1995), Minerva (2000 : 93-96) et Pellandra (2000 : 111-112) ont suffisamment attiré l'attention sur ce point, qui faisait déjà partie des idées de Rollin :

Il faut, s'il se peut, que l'utilité et l'agrément s'y trouvent ensemble afin que cette lecture ait pour les jeunes gens un attrait qui la leur fasse désirer. Ainsi, les livres qui sont purement de piété doivent leur être rarement proposés que d'autres, de peur que le dégoût qu'ils en auront une fois conçu ne les suive dans un âge plus avancé. (Rollin, Traité des études, 1765 [1726], t. I : 17)

1. Le titre de l'ouvrage de Barthélemy explicite ce parti-pris: une idéologie nouvelle, concernant l'éducation dans son ensemble, est au cœur de ces réflexions et de ces propositions.

2. La formation linguistique ajoute un nouvel objectif, très pratique. On propose (cf. la Grammaire de Barthélemy) de façon centrale l'acquisition par les jeunes filles d'une orthographe correcte, et ce savoir-faire doit s'appuyer sur des connaissances phonétiques et prosodiques, morpho-syntaxiques et lexicales/sémantiques. La connaissance de la grammaire est ainsi considérée comme indispensable: mais il s'agit d'une grammaire simplifiée aux seuls buts de la maîtrise de l'orthographe. Cette constatation est la même que M. Cohen (1967), et plus tard A. Chervel (1977), feront quant à la grammaire scolaire du XIX ${ }^{\mathrm{e}}$ 
siècle : "Pour écrire correctement le français, il va donc falloir devenir grammairien » (Chervel $1977: 46$ ).

3. La simplification de l'étude grammaticale ne s'accompagne pas d'une exigence moindre quant à la «qualité » ou à la «rigueur» de la formation dispensée ou prétendue : au contraire, la maîtrise de l'orthographe doit être complète (autant l'orthographe d'usage que l'orthographe de principe, distinction que P. Restaut établit en 1732). On peut penser cependant que la gymnastique intellectuelle (mémorisation, analyse, réflexion...) n'est pas poussée à l'extrême, vu le cadre privé et élitaire où s'effectue cette formation.

Nous pouvons dire ainsi que les grammaires destinées aux dames constituent un maillon, voire un "laboratoire ", dans le processus de disciplinarisation de la langue française (cf. Chervel 2008 : 49-66). Elles préfigurent la grammaire scolaire du XIX ${ }^{\mathrm{e}}$ siècle, quant aux objectifs de formation dans les deux "niveaux » de scolarisation : l'orthographe pour les petits, l'accès à la littérature (et donc, la formation du goût et du style) pour les adolescents. Mais avec des pertes notables, concernant notamment les habiletés langagières orales, la formation du « cœur » et, bien sûr, la pédagogie souple qui y était pratiquée.

\section{BIBLIOGRAPHIE}

AYRES BENNETT, Wendy éd. (1994a). « La grammaire des dames. Women's grammar », Histoire Épistémologie Langage, 16/II.

AYRES BENNETT, Wendy (1994b). « Le rôle des femmes dans l'élaboration des idées linguistiques au XVII ${ }^{e}$ siècle en France ", Histoire Épistémologie Langage, 16/II, 35-53.

BECK-BUSSE, Gabriele (1994a). « La grammaire française dédiée à mes jeunes amies : bibliographie raisonnée de manuels de la langue française à l'usage de la jeunesse féminine (1564-1850) », Histoire Épistémologie Langage, 16/II, 9-33.

BECK-BUSSE, Gabriele (1994b). «Les 'femmes' et les 'illitterati' ; ou : la question du latin et de la langue vulgaire », Histoire Épistémologie Langage, 16/II, 77-94.

BESSE, Henri (1996). «Traduction interlinéaire et enseignement des langues (chez Locke, Du Marsais, Radonvilliers, Robertson, et quelques autres ", Documents pour l'histoire du français langue étrangère ou seconde, 18, 293-312.

CHERVEL, André (1977).... et il fallut apprendre à écrire à tous les petits Français. Histoire de la grammaire scolaire. Paris : Payot.

CHERVEL, André (2008). Histoire de l'enseignement du français du XVII ${ }^{e}$ au XXe siècle. Paris : Retz.

CHEVALIER, Jean-Claude (1968). Histoire de la syntaxe [...] (1530-1750). Genève : Droz. Rééd. 2006.

CHEVALIER, Jean-Claude (1996). Histoire de la grammaire française. Paris : PUF.

COHEN, Marcel (1967). Histoire d’une langue, le français. Paris : Éditions sociales. $1^{\text {ère }}$ éd. 1947.

COMENIUS, Jan Amos ([1648] 2005). Novissima linguarum methodus. La toute nouvelle méthode des langues. Genève-Paris : Librairie Droz. Édition bilingue. 
FERNÁNDEZ FRAILE, Ma Eugenia (2011). « La educación lingüística de las mujeres », in J. Villoria éd., Historia de las ideas lingüísticas. Francfort : Peter Lang, 91-121.

FÉNELON, François (1883-1909 [1687]). L'Éducation des filles. Paris : Librairie Ch. Delagrave.

FLEURY, Abbé (1687). Traité du choix et de la conduite des études. Paris : Chez Pierre Aubouin.

LAMBERT, Madame de (1728). Avis d'une mère à sa fille. Paris : Ganeau.

MINERVA, Nadia (2000). « Le donne e la grammatica. Su alcune 'grammaires des dames' tra Sette e Ottocento ", in Nadia Minerva éd., Dames, demoiselles, honnêtes femmes, Bologne : CLUEB, 73-105.

PELLANDRA, Carla (2000). « La grammatica delle dame », in Lionello Sozzi (dir.), L'educazione dell'uomo e della donna nella cultura illuministica. Atti del Convegno Internazionale di Torino,1997, Turin : Accademia delle scienze, vol. 24.3, 103-115.

REUILLON-BLANQUET, Madeleine (1994). « Les grammaires des dames en France \& l'apprentissage des langues à la fin du XVIII ${ }^{\mathrm{e}}$ siècle [...] », Histoire Épistémologie Langage, 16/II, 55-76.

REUILLON-BLANQUET, Madeleine (1995). « Grammaires des Dames, 1770-1815. Ou comment rendre 'instructive et amusante' une étude considérée comme rébarbative », in Langages de la Révolution (1770-1815). Actes du 4 e colloque international de lexicologie politique, Saint-Cloud, Hauts-de-Seine, 163-175.

\section{NOTES}

1. Nous citons l'édition de Paul Rousselot de 1883 (cinquième édition en 1909).

2. Notre choix du corpus s'appuie sur les études de Beck-Busse (1994) et Minerva (2000). Pour les sources primaires citées, consulter le répertoire établi par $\mathrm{M}^{\mathrm{a}}$ Eugenia Fernández Fraile dans l'article publié dans ce même numéro. Nous réservons le terme 'manuel' aux ouvrages qui répondent à des programmes préétablis, et donc lorsque la matière objet d'étude est devenue une discipline scolaire (le XIX ${ }^{\mathrm{e}}$ siècle pour le cas du français langue maternelle).

3. Nous limitons la présentation de cette grammaire à quelques traits essentiels : Minerva (2000 : 80-86) s'en occupe de façon plus complète.

4. N'oublions pas que $\mathrm{Cl}$. Lancelot publie en 1663 , comme complément des méthodes de langues, quatre traités de poésie (latine, française, italienne, espagnole).

5. Nous n'introduisons pas la distinction entre français langue maternelle et français langue étrangère, car les deux catégories d'« apprenants » qu'elle présuppose n'était point totalement acquise encore, ni les 'grammaires' ne peuvent être départagées de façon stricte selon ce critère (cf. Chervel $2008: 42-43$ ).

6. Grammaire françoise, dans un goût nouveau, réduite en tables à l'usage des Dames et des autres personnes qui ne savent pas de latin. Ouvrage très utile aux demoiselles françoises qui enseignent cette langue en Allemagne. Berlin: Bei Ambrosius Haude und John Carl Spener. Nous n'avons pu consulter que le volume 1 de l'édition de 1747. La page de titre reproduit le titre en bilingue français-allemand.

7. L'auteur y fait référence dans l'Avis des Quatre Chapitres. Nous n'avons pu consulter que la seconde édition, de 1786.

8. Adjectif qui doit être pris dans son sens traditionnel: «qui appartient à l'amour, qui en procède » (Dictionnaire de l'Académie, 1762). 


\section{RÉSUMÉS}

Les Grammaires des dames effectuent un choix parmi les qualités langagières qu'elles doivent cultiver chez les dames, pour atteindre les finalités éducatives qui sont " propres à leur condition ", dont des vertus morales, bien sûr. À travers l'examen d'une série de grammaires destinées aux dames publiées au XVIIIe siècle (celles de : Antonini, Choffin, De Prunay, Panckoucke, Barthélemy, Adam..., notamment), nous allons ainsi nous poser les questions suivantes : quelle formation linguistique est proposée aux dames ? quelles qualités langagières doivent-elles cultiver ? Mais aussi, et en conséquence, quelles adaptations dans l'étude de la grammaire sontelles faites ? Nous découvrons ainsi que les grammaires des dames constituent un maillon essentiel dans la disciplinarisation de la langue française : elles préfigurent la grammaire scolaire $\mathrm{du} \mathrm{XIX}^{\mathrm{e}}$ siècle, quant aux objectifs de formation dans les deux « niveaux " de scolarisation : l'orthographe pour les petits, l'accès à la littérature pour les adolescents (et donc, la formation du goût et du style).

The Grammars of the ladies make a choice among the linguistic qualities which these have to cultivate, to reach the educational purposes which are "appropriate for their condition", among them the moral virtues, of course. Through the examination of a series of grammars intended for the ladies published in the 18th century (those of Antonini, Choffin, De Prunay, Panckoucke, Barthélemy, Adam...), we are so going to ask the following questions: what linguistic training is proposed to the ladies? What linguistic qualities do they have to cultivate? But also, and consequently, what adaptations in the study of the grammar are made? We discover that the grammars of the ladies constitute an essential link in the disciplinarisation of the French language: they prefigure the school grammar of the $19^{\text {th }}$ century, as for the objectives of training in both levels of schooling: the spelling for young people, the access to the literature for the teenagers (and thus, the training of the literary taste and the style).

\section{INDEX}

Mots-clés : Grammaire des dames, Éducation linguistique, Disciplinarisation du français, Grammaire scolaire, Enseignement de l'orthographe, Textes littéraires

Keywords : Grammar of the ladies, Linguistic education, Disciplinarisation of French language, School grammar, Teaching of the spelling, Literary texts

\section{AUTEUR}

\section{JAVIER SUSO LÓPEZ}

Université de Grenade 